\title{
Linx
}

Revue des linguistes de l'université Paris X Nanterre

$77 \mid 2018$

Regards croisés sur le futur en français et dans différentes langues romanes

\section{Retour morphosémantique sur les futurs irréguliers de l'espagnol}

Eric Beaumatin et Élodie Blestel

\section{CpenEdition}

Journals

Édition électronique

URL : http://journals.openedition.org/linx/2826

DOI : 10.4000/linx.2826

ISSN : 2118-9692

Éditeur

Presses universitaires de Paris Nanterre

Édition imprimée

Date de publication : 30 octobre 2018

Pagination : 159-184

ISBN : 978-2-84016-329-9

ISSN : 0246-8743

Référence électronique

Eric Beaumatin et Élodie Blestel, « Retour morphosémantique sur les futurs irréguliers de l'espagnol », Linx [En ligne], 77 | 2018, mis en ligne le 30 avril 2019, consulté le 03 mai 2019. URL : http:// journals.openedition.org/linx/2826; DOI : 10.4000/linx.2826

Ce document a été généré automatiquement le 3 mai 2019.

Département de Sciences du langage, Université Paris Ouest 


\title{
Retour morphosémantique sur les futurs irréguliers de l'espagnol
}

\author{
Eric Beaumatin et Élodie Blestel
}

\section{La question des formes du futur (et du conditionnel) en espagnol}

1 La littérature abonde au sujet des valeurs (temporelles, modales voire aspectuelles) des futurs et des conditionnels dans les langues romanes, de l'alternance de formes synthétiques vs analytiques (ou simples vs périphrastiques) comme de leurs contraintes syntactico-sémantiques de construction ${ }^{1}$. Elle est étonnamment plus modeste s'agissant de leurs formes variées, singulièrement en ce qui concerne l'espagnol. On peut dès lors se demander en quoi les formes «irrégulières » des futurs synthétiques du verbe espagnol (p. ex. VENIR $\rightarrow$ vendré alors que CANTAR $\rightarrow$ cantaré) constituent un objet d'étude pertinent en soi, ou ce que leur étude peut apporter de spécifiquement intéressant. Encouragés par quelques notables précédents ( $c f$. infra $\S 3$ ), et compte non tenu de quelques extensions problématiques de la question apparues chemin faisant ${ }^{2}$, disons qu'il s'agit de se demander si la morphologie irrégulière de certains de ces futurs présente des sub-régularités paradigmatiques d'échelle qui en feraient un sous-ensemble cohérent de futurs marqués dans le système, corrélativement à un comportement syntaxique particulier et/ou des propriétés (ou affinités) sémantiques particulières. En somme de voir, si, saisies en synchronie dans la langue d'aujourd'hui, ces irrégularités peuvent être tenues pour relativement motivées au sens de la leçon saussurienne que retient M. Launay (2003: 278) ; si oui, en quoi et de quelle façon; de voir, enfin, s'il y a moyen de dégager une sémiologie spécifique à ce sous-ensemble.

2 Car l'on doit en effet poser, par hypothèse, qu'il y a en espagnol des futurs irréguliers. Et c'est à partir des traits morphologiques de leur(s) irrégularité(s) que nous en aborderons l'examen, pour des questions et propositions les concernant en propre comme intéressant l'analyse morphologique et submorphémique du futur espagnol en général ${ }^{3}$. 


\section{Les traits constitutifs d'irrégularité morphologique dans un système verbal}

3 En matière de formes verbales dans les langues romanes, G. Boyé (2000:27-32) rappelle qu'elles peuvent porter sur :

- La flexion : esp. indic. prés. P1 canto (CANTAR) / doy (DAR)

- Le radical : esp. indic. prét. P1 canté (CANTAR) / tuve (TENER)

- Des faits de supplétion : esp. IR, P1 : fui (indic. prét.) / iré (indic. fut.)

- Des «variations» paradigmatiques : de radical (ex. fr. ASSEOIR indic. prés. P3 :

assied / asseoit) ou de groupe (ex. ital. AMMOLLIRE / AMMOLLARE)

4 On notera qu'à assumer ces catégories, le futur espagnol ne connaît pas de flexions déviantes ${ }^{4}$, ni n'est touché par le phénomène de supplétisme ${ }^{5}$. La question de la variation paradigmatique peut être posée et le sera plus loin (§ 4). À strictement parler, le radical n'est pas non plus touché dans ces futurs ${ }^{6}$.

Qu'est-ce que le linguiste (mais aussi peut-être le locuteur) peut donc être amené à appeler « irrégulier » ? Ou du moins à repérer comme tel, s'agissant d'une forme de futur en espagnol ? On constate que deux réponses possibles sont traditionnellement données, que nous recevons volontiers en première approximation :

- Sur le versant diachronique, tout ce qui ne répond pas strictement au schéma de formation des futurs et conditionnels : \{infinitif complet du verbe + désinence de HABER (à l'indic. prés. / resp. à l'indic. imp.) $\}^{7}$

- Sur le versant synchronique, tout ce qui ne permet pas de reconnaître pleinement l'infinitif dans un futur, donc de l'y associer paradigmatiquement (i.e. analogiquement, pour la lecture qu'en fait le locuteur), ainsi : PONER $\rightarrow$ pondré, ou $\mathrm{HABER}^{8} \rightarrow$ habré, ou encore QUERER $\rightarrow$ querré, alors que, rappelons-le : CANTAR $\rightarrow($ cantar + he =) cantaré.

L'esprit d'une telle assignation d'irrégularité mérite commentaire, en ce qu'elle pourrait comporter un biais consistant à la faire reposer sur un préalable de description synchronique passablement verrouillé a priori, c'est-à-dire sur le postulat que l'infinitif serait le composant de référence pertinent dans l'analyse morphologique des formes du futur. Ou, en d'autres termes, à rabattre la raison de l'analyse synchronique de ces formes sur un postulat diachronique non quelconque concernant leur mode de formation : là est précisément, comme on le verra, l'un des enjeux de ce travail.

\section{Le traitement de ces irrégularités}

7 On peut donc se contenter de leur trouver une "explication" restrictivement diachronique : c'est la position résolument assumée par beaucoup, dont la RAE dans son Esbozo, au terme de longues tergiversations: «Il est préférable d'expliquer historiquement la formation du futur et du conditionnel, [...] sans entrer dans les détails » 9 .

8 C'est la conclusion non moins résignée - en quelque sorte faute de mieux - à laquelle se rend G. Luquet, notamment découragé par la résistance des formes VALER $\rightarrow$ valdré et SALIR $\rightarrow$ saldré :

Il n'existe pas de réponse satisfaisante à cette question. Et il n'est peut-être pas nécessaire d'en chercher une, car si un linguiste est dans son rôle lorsqu'il cherche à comprendre pourquoi les formes d'une langue sont ce qu'elles sont [...], il 
outrepasse les limites de sa compétence lorsqu'il prétend expliquer pourquoi ces formes ne sont pas ce qu'elles pourraient être. (Luquet 2000a : 70). J. W. Harris, en raison de l'incapacité qu'il estime déceler chez ce dernier à se dégager du préjugé de prégnance formelle de l'infinitif au niveau des composants morphologiques du futur, alors que J. W. Harris lui-même, en l'occurrence plus phonologue que morphologue, proteste expressément de son souci synchroniste (Alcoba 1992 : 37). (non nécessairement exclusifs), à commencer par les projets que l'on pourrait qualifier d'intégrationnistes: ces derniers visent à rendre compte de la formation de tous les futurs (ici espagnols, réguliers et irréguliers ensemble) au moyen d'un seul et même modèle, aussi performant que possible, i.e. susceptible de ne laisser de côté qu'aussi peu de scories que possible ${ }^{10}$, en intégrant en profondeur toutes les formes pour reléguer les irrégularités à des manifestations de surface. L'enjeu est de proposer une morphophonologie efficace et sans a priori (normatif ou diachronique). En l'espèce, s'agissant des futurs irréguliers espagnols, et dans la mesure où l'infinitif n'est par exemple plus intégralement repérable dans PONER $\rightarrow$ pondré, il était tentant de se rabattre sur le radical (ici pon-, à gauche de la voyelle thématique) pour élargir le rendement du modèle. La plupart des modèles ambitieux (notamment d'inspiration générativiste ou affins) tournent autour d'une proposition de ce type ${ }^{11}$ :

Une autre stratégie, que l'on pourrait au contraire qualifier de provisoirement désintégrationniste (donc, une fois de plus, et comme cela apparaitra, non exclusive du souci des précédentes) consisterait à rechercher plutôt, quoique toujours en synchronie, $\mathrm{si}$, dans le système verbal espagnol, le sous-ensemble de ces formes irrégulières est redevable d'une sémiologie propre, spécifique, différentielle d'avec les formes régulières.

\section{La quête d'une sémiologie spécifique}

Ces formes irrégulières du futur espagnol que l'on tiendrait dès lors pour marquées, à quoi les associer sémiologiquement de syntaxique ou sémantique?

Les glorieux précédents de pareille entreprise ne manquent pas dans le cadre théorique du guillaumisme ou du post-guillaumisme, notamment hispaniste en France, à partir de questions du même type posées (on peut dire désormais «traditionnellement » à certaine échelle épistémologique ${ }^{13}$ ) aux formes irrégulières du prétérit, en l'état synchronique de l'espagnol actuel. Ils en constituent même comme une marque de fabrique ${ }^{14}$. On s'est 
d'abord surtout intéressé à la poignée de parfaits dits «forts » (derniers héritiers en cela de paradigmes latins ainsi désignés), qui cumulaient non seulement toute une série de modifications vocaliques et consonantiques du radical à toutes les personnes, mais également le trait d'une accentuation rhizotonique à P1 et $\mathrm{P} 3$, alors qu'elle porte sur la flexion aux formes régulières de ces personnes : irr. TENER $\rightarrow$ P1 tuve alors que rég. TEMER $\rightarrow$ P1 temí. Cet ensemble a été mieux précisé par G. Luquet sous le vocable de prétérits "irréguliers ", afin d'y intégrer notamment les formes monosyllabiques SER $\rightarrow$ fui et DAR $\rightarrow$ di qui, en toute rigueur, ne peuvent être tenues pour fortes.

Indépendamment de diverses hésitations notionnelles entre syntaxe et sémantique pour trouver et formuler la commune raison de leur commun trait d'irrégularité (verbes existentiels, puissanciels, modaux ; verbes désémantisés ; verbes sémantiquement frappés de certaine "antériorité mentale"; verbes auxiliarisables, etc.), on tourne là autour d'une intuition robuste: dans toutes les langues où se produit et se laisse observer ce phénomène (langues romanes, mais c'est aussi le cas de l'anglais), il s'agit incontestablement de verbes spécialement fréquents, dont on ne saurait se passer dans l'usage courant : des verbes en tout état de cause «fondamentaux ». Toute la question est de savoir à quel titre ils sont fondamentaux, au-delà de la simple constatation de leur remarquable fréquence. C'est un peu le nœud de la question.

En effet, cette question posée en synchronie est cependant instruite d'information diachronique: tous ces parfaits irréguliers ne constituent aujourd'hui que le reliquat assez stable d'un plus vaste ensemble dont la plupart des éléments, forts depuis le latin, ont progressivement été pris dans un mouvement de réfections analogiques qui les a « régularisés » $:$ SCRIBERE $\rightarrow$ scripsi > ESCRIBIR $\rightarrow$ escripse, mais ensuite escribí. Pourquoi certains, comme SABER, PODER et quelques autres ( $c f$. infra, notre tableau du §5) ont-ils résisté ? Pourquoi ceux-là et pas les autres? Qu'est-ce qui les a protégés de la réfection régularisatrice? C'est à ces interrogations que la notion de verbes fondamentaux cherche à répondre en s'affinant de diverses manières. On ajoutera ces deux observations décisives que : a) ces verbes n'ont pourtant pas été complètement insensibles à l'influence des formes régulières puisque le timbre vocalique distinctif de leur désinence personnelle, même atone à $\mathrm{P} 1$ et $\mathrm{P} 3$, s'est aligné sur elles (resp. en -e et -0 ) ; b) une tendance régularisatrice est par ailleurs nettement patente à l'échelle de ce sousensemble, par exemple en la réduction du nombre de voyelles radicales: de $a / o / i / u$ encore possibles au Moyen-âge, $i / u$ seuls ont prospéré ${ }^{15}$, mais demeurent très différents des paradigmes réguliers: cette concentration des marques d'irrégularité est plus suggestive encore de sa corrélabilité à une sémiologie spécifique.

Sur cette lancée, la même question n'a pas manqué d'être posée aux formes irrégulières du présent (de l'indicatif comme du subjonctif ${ }^{16}$ ) ou des participes passés (Toledo 2013), et peut légitimement se poser pour les formes de futurs irréguliers: c'est ce qu'a tenté Luquet (2000a: 61-70) en finissant par jeter le gant (on verra plus loin devant quels obstacles).

\section{Questions de périmètre}

23 Le périmètre des verbes candidats à être considérés comme irréguliers dépendra des phénomènes (alternances consonantiques, vocaliques, diphtongaisons ou autres apophonies, associées ou non à des déplacements d'accent) que l'on estimera constituer des traits isolément ou conjointement caractéristiques de cette irrégularité, donc de la 
définition même que l'on donnera à l'irrégularité en question. Mais ces traits ne sauraient se suffire à eux-mêmes et la survenue (ou la stabilité) de ces phénomènes est parfois vacillante.

Ainsi, malgré le postulat normatif selon lequel les composés par préfixation de ces verbes irréguliers subissent les mêmes irrégularités que les formes de référence ( $v \cdot g$. au prétérit TENER $\rightarrow$ tuve, donc DETENER $\rightarrow$ detuve), on peut découvrir des attestations de formes concurrentes, par exemple celle de CONTRAER $\rightarrow \mathrm{P} 4{ }^{*}$ contraímos (au lieu de norm. contrajimos, sur TRAER $\rightarrow$ trajimos) ... dans la bouche même du Président en exercice de la Real Academia Española ${ }^{17}$ : outre que la saturation sémantique et la fréquence intrinsèques de CONTRAER varient en raison inverse de celles de TRAER ${ }^{18}$, il y des chances pour que l'association morphosémantique de l'un à l'autre soit relativement faible dans l'esprit du locuteur. De même, à considérer que le degré de saturation sémantique (ou, du moins, dans l'usage, la spécification/restriction sémantique en discours), ou encore d'autres paramètres (auxiliarisabilité, etc.) soient en cause, on ne s'étonnera pas, selon cette logique, que lorsque ANDAR est utilisé comme auxiliaire ou en construction attributive, sa forme de prétérit P3 anduvo soit stable, alors que la forme faible aujourd'hui tenue pour très anti-normative *andó survient prévisiblement lorsque ce verbe est, en telle occurrence ponctuelle, saisi dans son acception parasynonymique de CAMINAR ('marcher') : ce sont là autant de confirmations a contrario de la pertinence de l'hypothèse d'une sémiologie spécifique à ces sous-ensembles de verbes marqués d'irrégularité(s). Là fait sans doute retour la question de la " variation » au sens de Boyé ( cf. supra, § 2) mais, à travers de telles variantes, on se retrouve surtout devant une précieuse occasion d'observer en direct les tensions évolutives de la langue.

De même au futur, et pour des raisons similaires, certains composés verbaux par préfixation se comportent-ils de façon sinon erratique du moins hésitante dans les faits, ou encore selon des affinités variables : si DESHACER $\rightarrow$ deshará ou REHACER $\rightarrow$ rehará (et même SATISFACER $\rightarrow$ satisfará) suivent les irrégularités de HACER $\rightarrow$ hará, ce n'est pas le cas de la série PRE-/BEN-/MAL-/DES-DECIR, qui présentent des futurs réguliers en predeciremos etc., malgré DECIR $\rightarrow$ diremos (et même CONTRADECIR $\rightarrow$ contradiremos) ${ }^{19}$. C'est dire, là encore, qu'à la mesure de l'éloignement morphosémantique de ces composés d'avec leur base, et en phase avec leur plus grande saturation sémantique intrinsèque, décroit compréhensiblement la pertinence de leur marquage d'irrégularité comme signifiant de quelque statut « fondamental ».

Mais il convient surtout de noter que, si le comportement morphologique de certains verbes peut paraître à tel ou tel égard irrégulier en tel tiroir verbal (sont basiquement concernés: prétérit [et subjonctifs en -ra, -se et -re], présent [de l'indicatif et du subjonctif], futur [et conditionnel], participe passé, impératif), il peut ne pas l'être dans le cadre de tel autre. Ainsi ANDAR, irrégulier au prétérit, ne l'est-il pas au futur ni au présent, et VALER ${ }^{20}$ ou SALIR, irréguliers au futur, ne le sont-ils pas au prétérit.

Que le périmètre des verbes irréguliers soit éventuellement un peu flou, au futur comme en d'autres tiroirs verbaux, conforte (plus qu'il ne la ruine) la pertinence de l'hypothèse de raisonner sur leur spécificité en tant que verbes «fondamentaux ", quelle que soit la précision qu'il reste à donner à cette idée. De plus, que ce périmètre diffère selon le tiroir verbal considéré, plutôt que de nous décourager dans cette quête d'une sémiologie spécifique à ces morphologies irrégulières, suggère tout l'intérêt qu'il y aurait à affiner l'hypothèse d'une notion syntactico-sémantiquement variable de ce caractère 
«fondamental» en fonction des propriétés et contraintes non quelconques et différentielles du tiroir verbal concerné.

Observons maintenant ces morphologies :

\section{5. État des lieux morphologique et premiers éléments de sémiologie}

\begin{tabular}{|c|c|c|c|c|c|}
\hline & & FUTUR & Prétérit & Présent ind. & Impératif \\
\hline \multirow{3}{*}{ Formes régulières } & Cantar & CANTARÉ & CANTÉ & CANTO & CANTA \\
\hline & Comer & COMERÉ & COMÍ & сомо & COME \\
\hline & Partir & PARTIRÉ & PARTÍ & PARTO & PARTE \\
\hline \multirow{2}{*}{$\begin{array}{l}\text { Infinitif à } \\
\text { radical contracté }\end{array}$} & Decir & diré & dije & digo & $d i$ \\
\hline & Hacer & haré & hice & hago & haz \\
\hline \multirow{5}{*}{$\begin{array}{l}\text { Chute de la } \\
\text { prétonique interne }\end{array}$} & Caber & cabré & cupe & quepo & CABE \\
\hline & Haber & habré & hube & he & \\
\hline & Poder & podré & pude & PUEDO & PUEDE \\
\hline & Querer & querré & quise & QUIERO & QUIERE \\
\hline & Saber & sabré & supe & sé & SABE \\
\hline \multirow{5}{*}{$\begin{array}{l}\text { Chute vocalique } \\
\text { et épenthèse } \\
\text { consonantique }\end{array}$} & Poner & pondré & puse & pongo & pon \\
\hline & Salir & saldré & SALÍ & salgo & sal \\
\hline & Tener & tendré & tuve & tengo & ten \\
\hline & Valer & valdré & VALÍ & valgo & val/VALE \\
\hline & Venir & vendré & vine & vengo & ven \\
\hline \multirow{5}{*}{$\begin{array}{l}\text { Pas d'irrégularité au futur } \\
\text { (contraintes phonétiques diverses) }\end{array}$} & Ir & IRÉ & fui & voy & ve \\
\hline & Ser & SERÉ & fui & soy & sé \\
\hline & Dar & DARÉ & $d i$ & doy & DA \\
\hline & Estar & ESTARÉ & estuve & estoy & ESTÁ \\
\hline & Traer & TRAERÉ & traje & traigo & TRAE \\
\hline
\end{tabular}




\begin{tabular}{|l|l|l|l|l|l|}
\hline -ducir & -DUCIRÉ & -duje & conduzco & -DUCE \\
\hline
\end{tabular}

Table 2. Table synoptique des irrégularités dans les différents paradigmes (En capitales, les formes non irrégulières)

Trois types d'irrégularités apparaissent :

1) Infinitif à radical contracté (type DECIR $\rightarrow$ diré)

2a) Chute de la prétonique interne (type CABER $\rightarrow$ cabré),

2b) Chute de la prétonique interne et épenthèse consonantique (type TENER $\rightarrow$ tendré)

Ce tableau appelle deux premières observations: a) l'irrégularité de ces futurs rend l'infinitif illisible dans sa forme pleine (diré n'est pas DECIR + he, et vendré n'est pas VENIR + he); b) dans toutes les formes irrégulières, on a perdu la voyelle thématique (comp. come-r-é vs pod-r-é)

-r- est commun à toutes les formes, régulières comme irrégulières. La question se pose de son statut, dès lors que, dans les formes irrégulières, il ne peut plus être lisible comme un morphème d'infinitif. À l'échelle de l'ensemble du paradigme du futur, on peut faire l'hypothèse d'une réanalyse, ce que les formes irrégulières permettent de mieux comprendre : de morphème - $r$ d'infinitif, une fois dissocié de cet infinitif dans les formes de futur, il est devenu -r-, morphème de futur. On peut donc suivre en cela Molho (1992), Azzopardi (2011) et Pagès (2012)21.

Si l'on admet que l'infinitif n'est plus le composant morphologiquement pertinent dans le paradigme du futur en synchronie, il est possible de poser que, dans la partie gauche des formes, c'est le radical, toujours lisible, qui le devient : suivi de - $r$ - + voyelle thématique + désinence dans les formes régulières; sans voyelle thématique dans les formes irrégulières ( $c f$. supra $\S 2$ ).

On a ainsi, dans le paradigme des futurs dans leur ensemble :

- d'une part des radicaux marqués

soit par la perte de la voyelle thématique

soit par par un radical différent du radical de l'infinitif (DECIR $\rightarrow$ diré, HACER

$\rightarrow \underline{\text { haré})}$

- d'autre part, des radicaux qui conservent le radical de l'infinitif et leur voyelle thématique.

Remarquons qu'en bien des cas (PONER, SALIR, TENER, VALER, VENIR), une consonne épenthétique apparaît (pondré etc.), toujours /d/. En tout état de cause, qu'il y ait eu chute de la voyelle thématique ou pas, suivie d'épenthèse de /d/ ou pas, toutes les formes de futur satisfont deux exigences simultanées : le radical est protégé et l'-r- de futur aussi.

\section{Comportement syntaxique et sémantique des verbes « fondamentaux »}

Après ces premières observations sémiologiques, nous pouvons observer que les formes irrégulières présentent également un comportement syntaxique singulier :

Nombre de ces verbes servent d'auxiliaires. M. Bénaben remarque que la plupart des formes irrégulières se prêtent à la fonction d'auxiliaire ou de semi-auxiliaire (2002:161). Si l'on écarte les propositions des auteurs pour lesquels est auxiliaire tout verbe qui s'est 
"désémantisé ", on peut s'en tenir à la proposition de Pottier (1961) qui propose de définir l'auxiliaire comme tout verbe incident à un autre verbe dans le même syntagme verbal, ou celle de M. Launay (1980) pour lequel l'auxiliarisation est un mécanisme qui repose sur l'altération de la valence d'un verbe, par la perte de son foncteur. Le changement ne serait plus sémantique, mais il concernerait les propriétés syntaxiques dudit verbe ${ }^{22}$. C'est le cas de presque tous les verbes irréguliers, à l'exception de DECIR ${ }^{23}$. Même VALER, CABER et SALIR ont tous trois leur place puisqu'ils présentent cette possibilité syntaxique ${ }^{24}$ :

(1) un modelo peculiar que cabe definir como municipalista.

[un modèle particulier qu'il y a lieu de définir comme municipaliste]

(2) ¿Quién sale ganando aquí ?

[Qui y gagne, ici ?]

(3) Más vale mancharse las manos de sangre que lavárselas.

[Plutôt que de s'en laver les mains, mieux vaut se les tacher de sang]

Ces verbes entrent dans des constructions attributives: même SALIR et VALER, qui faisaient obstacle au projet de Luquet, le permettent. En l'occurrence, le verbe "attributif» permet d'octroyer une propriété ou une identité (apport) à un support prédicatif. Les verbes d'état jouent ce rôle mais, contextuellement, certains verbes transitifs peuvent entrer dans ce type de construction: c'est le cas de l'exemple de M. Bénaben : « El coche me sale caro » (2002:161). Dans une construction de ce type, le verbe peut être suivi ${ }^{25}$ :

(4) d'un nom : «[...] cede en secreto la paleta al hijo que le salió artista prodigioso.»

[il cède secrètement sa palette au prodigieux artiste de fils qu'il a eu]

(5) d'un adjectif : « Si me llegan a poner el Esclavo me sale una birria, pero pusieron

el torso del Belvedere y me salió estupendo. »

[Si on m'avait refilé l'Esclave, j'aurais fait quelque chose de nul, mais on m'a donné le torse du Belvédère et ça a donné un truc formidable]

(6) d’un participe : « Paco Ordóñez salió encantado de la reunión »

[Paco Ordóñez est sorti ravi de la réunion]

(7) d'un syntagme prépositionnel : « el problema con la [carne] de guerra es que a veces sale con mucho plomo o con gases y nos da mucho más trabajo limpiarla »

[le problème avec la viande de guerre, c'est que parfois elle arrive pleine de plomb ou de gaz et que ça nous donne encore plus de travail pour la nettoyer]

Constructions impersonnelles : on peut aussi observer que certains verbes de cette liste entrent dans des constructions impersonnelles (même si l'on ne peut pas parler de verbes impersonnels per se comme pour llover, nevar, anochecer, etc. $)^{26}$ :

(8) Hay que vivir para saber morir

[Il faut vivre pour savoir mourir]

(9) No cabe afirmar, por otra parte, que las ciencias transdisciplinarias sean más importantes que las mono, bi, inter o multidisciplinarias.

[Il n'y a pas à affirmer, par ailleurs, que les sciences transdisciplinaires soient plus importantes que les mono, bi, inter ou multidisciplinaires]

(10) Bien se puede que le haya picado el virus del Barrio

[Il se peut bien qu'il ait été atteint par le virus du Quartier]

(11) ahora pues salió que el programa seguía siendo lo mismo

[et là, donc, il se trouve que l'émission était toujours la même chose]

(12) Y no vale que el sistema se lave las manos

[Et ce n'est pas juste que le système s'en lave les mains]

En d'autres termes, si VALER et SALIR posaient problème à G. Luquet pour définir au futur une catégorie de verbes "fondamentaux », en ce qu'ils ne sont pas irréguliers au prétérit en même temps qu'au futur, il convient de remarquer au moins que, quant au syntaxique et au sémantique, ils ne le cèdent en rien aux autres. Et, comme il a été dit plus haut (fin 
du § 4), peut-être les propriétés du futur devraient-elles intervenir dans une telle définition. De plus, ces traits syntaxiques ou sémantiques, tendanciellement cumulatifs, peuvent fort bien ne pas apparaître simultanément sur un verbe donné.

En revanche, et sauf à établir une hiérarchie a priori entre tiroirs verbaux au regard de ces irrégularités, il importe de noter que, à l'inverse, certains verbes irréguliers par ailleurs ne le sont pas au futur : IR, SER, DAR, ESTAR, TRAER et les verbes en -DUCIR. Les verbes en -DUCIR, assez saturés sémantiquement, non auxiliarisables ni susceptibles de construction attributive, s(er)ont vraisemblablement, avec TRAER et ses préfixés, les premiers candidats à se régulariser à terme au prétérit (CONDUCIR $\rightarrow{ }^{*}$ conducí alors que norm. conduje) sous la forte pression de l'usage, resserrant ainsi à cette échelle paradigmatique la validité de l'hypothèse des « verbes fondamentaux » : on ne s'étonnera pas qu'au futur ils ne soient pas irréguliers. Quant aux autres, on avancera que les verbes monosyllabiques IR, SER et DAR posant des problèmes particuliers en ce qu'ils présentent des sommets vocaliques à la fois radicaux et thématiques, ils se prêtent peut-être moins aisément aux réfections régularisantes. Nous joignons à cette liste de verbes monosyllabiques le verbe ESTAR, dont le $e$ - prothétique n'est autre qu'un élément d'appui phonétique $^{27}$, comme le souligne D. Zalio :

Seul verbe oxytonique de la conjugaison espagnole, le verbe estar est contre toute apparence un monosyllabe. D'une part, du seul point de vue de la conjugaison espagnole, il partage avec les verbes monosyllabiques dar, ir et ser la présence d'un yod, créant un suffixe dit " protecteur " à la première personne du singulier du présent de l'indicatif [...]. D'autre part, l'inclusion d'estar dans ce réseau quadripartite est justifiée par l'impossibilité pour les formes espagnoles de posséder un $s$ en position initiale suivi d'une consonne, impossibilité palliée par le recours à la prothèse d'un /e/ (espada, escepticismo, estación, estrella, etc.). Ce /s/ joue donc un rôle purement phonétique. (Zalio $2013: 196)^{28}$

Reste que ceux qui sont irréguliers au futur peuvent sans mal se confirmer dans leur statut de verbes "fondamentaux", sous réserve d'en affiner les conditions définitoires, notamment au futur: désémantisation (ou faible saturation sémantique) donc subduction, possible préséance entre critères syntaxiques et sémantiques au-delà de leur simple concomitance, degrés de "fondamentalité » (existenciels, puissanciels, modaux etc.), critère de l'«antériorité mentale» (encore en attente de précision)... Quelque moyen que l'on se donne pour formuler au mieux ce qui, de leurs particularités syntactico-sémantiques est à corréler au marquage morphologique qui les affecte, et sans s'inhiber dans cette attente, on peut proposer d'autres voies de recherche, attentives à la ténuité qu'ils laissent apparaître de la limite entre lexique et grammaire.

\section{Le morphologique et son en-deçà}

En effet, lorsqu'elles s'opposent aux formes grammaticales, on a coutume de définir les formes lexicales comme (i) des unités qui appartiennent à un ensemble ouvert (contrairement aux grammèmes qui constituent un ensemble fermé), et (ii) comme un ensemble de morphèmes sémantiquement dénotatifs (quand les grammèmes servent seulement à mettre des éléments en relation ou établir des catégories) ${ }^{29}$. Or si l'on admet qu'il existe dans la langue un continuum entre lexique et grammaire ${ }^{30}$, nous observons que les verbes concernés par ces irrégularités (y compris VALER, SALIR et CABER, de plein droit au futur : cf. supra) sont plus « grammaticaux » que les autres, en ce sens qu'ils ne se 
limitent pas à leur fonction dénotative (renvoi à des concepts, à des choses du monde), ils jouent également un rôle relationnel et/ou catégorisant.

Par ailleurs, l'examen de la sémiologie des formes irrégulières du futur invite à reposer la question de la place et du statut du morphème $-r$ - \{futur\}. Nous avons montré que l'infinitif ne peut être considéré comme le composant pertinent du futur en synchronie et qu'il a fait l'objet d'une réanalyse : dissocié de l'infinitif, il opère, seul, comme morphème de futur, dans toutes les formes du paradigme (régulières comme irrégulières).

Or, en diachronie, nous pourrions avancer une hypothèse explicative à ce processus de réanalyse du morphème $-r$ - \{futur\} en le corrélant à la présence d'un opérateur caractéristiquement submorphémique ${ }^{31}$ dans un ensemble transparadigmatique plus large.

En effet, comme nous l'avons exposé précédemment, la linguistique du signifiant entend étudier ce que les relations d'analogie et de paronymie entre les divers signifiants permettent de déduire sémasiologiquement des relations entre les signifiés qui leur sont associés. La cognématique, élaborée par Bottineau au début des années 2000, permet d'aller encore plus loin, dans le sens de la compréhension des phénomènes d'iconicité entre propriétés articulatoires et amorçage du sens : elle propose d'isoler des composants submorphémiques qui entrent dans la composition des signifiants de nombreux soussystèmes grammaticaux et qui peuvent expliquer en partie ces processus de réanalyse. Ces composants, appelés "cognèmes ", véhiculent des instructions cognitives partiellement motivées par le profil sensori-moteur des phonèmes auxquels ils sont associés. Toutefois, la relation entre un phonème et un invariant cognitif (ou cognème) ne s'active que dans certaines circonstances (non cumulatives) :

1) il se manifeste dans une alternance récurrente [...]. 2) L'opérateur-mot dans lequel il se manifeste est lui-même globalement formé d'une agglutination de marqueurs extraits de telles alternances [...]. 3) Le submorphème, combiné à une racine ou à d'autres submorphèmes dans une position constante comme l'initiale ou la finale, classe tous les opérateurs concernés dans une catégorie donnée [...]. (Bottineau $2004: 29)^{32}$.

Pour la langue espagnole, plusieurs auteurs ont mis en évidence l'existence d'un cognème $\mathrm{R}$, qu'ils associent à l'invariant cognitif d'agentivité (Bottineau 2007 : 61, Luquet 2010 : 74, Quitard 2010 : 162, Pagès 2012 : 47, Fortineau-Brémond, à paraitre). Celui-ci émerge par iconicité avec l'une des propriétés articulatoires de $/ \mathrm{r} / \sim / \varsigma /$, à savoir, l'impulsion rendue nécessaire par le franchissement de la langue qui s'oppose au passage de l'air expiré :

Lors de l'émission d'un [r], par exemple, l'organe actif de l'articulation que constitue la langue fonctionne comme un obstacle qui s'oppose au passage de l'air et le franchissement de cet obstacle nécessite une augmentation sensible de l'air expulsé. (Luquet $2010: 74$ ).

44 C'est ce rapport iconique entre impulsion articulatoire de $/ \mathrm{r} / \sim / \Gamma /$ et sa présence dans divers sous-systèmes grammaticaux sémantiquement affins qui nous invite à reconnaître derrière ces phonèmes un cognème $\mathrm{R}$, lequel opère dès lors à la fois dans l'émergence de l'infinitif mais aussi dans celui du signifié du futur.

On peut alors céder à la tentation de voir dans le -r d'infinitif espagnol, et donc dans le signifié de cette forme verbale, l'une des réalisations possibles du cognème d'impulsion, et ce dans la mesure où un infinitif est associé à la notion d'« "agentivité puissantielle", ou pour employer une autre terminologie, la représentation d'une "opérativité 
puissantielle", celle du déroulement perspectif d'une opération associée à un agent dont l'identité formelle n'est pas définie » (Luquet, Ibid.) $)^{33}$

Ce même cognème intervient encore dans le signifié de la préposition por-préposition dont on posera qu'elle constitue un support de transmission entre deux entités en mouvement (Blestel et Fontanier 2017 : 197) $)^{34}$ - mais aussi dans les noms d'agents et d'instruments qui présentent un suffixe en -or, ainsi que dans la forme en - $r a^{35}$. Dans tous ces paradigmes, ce cognème $\mathrm{R}$ entre ainsi dans deux types de systèmes oppositifs. Il s'oppose à la fois au cognème $\mathrm{S}$ de 'continuation' quand il permet d'opposer les deux formes de subjonctif d'imparfait (forme en -ra et forme en -se) comme le montre Luquet (2010: 74), mais il s'oppose aussi au cognème $T$ qui véhicule un invariant cognitif de 'limite', comme l'a exposé Bottineau (2010: 30). Cette dernière paire oppositive est particulièrement visible dans le système verbal espagnol, notamment dans ce qui oppose les formes du monde non-personnel cantar et cantado, où le cognème d'impulsion' $R$ participe à la conceptualisation d'une virtualité dans l'infinitif, quand le cognème $\mathrm{T}^{36} \mathrm{de}$ 'limite' renvoie à un point de vue globalisant et rétrospectif sur le procès avec le participe (Blestel 2015). De la même manière, dans le système prépositionnel, por entre en concurrence avec des prépositions qui contiennent le cognème $\mathrm{T}$, notamment la préposition de dans les constructions passives pour lesquelles la préposition introductive varie selon qu'il s'agit d'une conception dynamique ou statique du complément d'agent (Blestel et Fontanier 2017 : 196).

47 Cette lecture cognématique de la sémiologie du futur n'en interdit pas la lecture morphologique : il s'agit là d'un niveau d'analyse supplémentaire, qui permet d'élaborer des hypothèses sur la façon dont se structure le tissu sémiologique de l'espagnol dans une optique transparadigmatique, tout en rétablissant le "chaînon manquant » entre la dimension phonatoire et la dimension sémantique du langage (Le Tallec-Lloret, $2012: 27$ ).

Notons par ailleurs que si ce cognème opère seul dans la morphologie du futur, il est nécessairement associé à une voyelle thématique dans le morphème d'infinitif car, pour reprendre les termes de Luquet, il n'y a "qu'un seul "morphème" d'infinitif en espagnol, celui qui est constitué de "la" voyelle thématique du verbe -quelle qu'elle soit- et d'un -r terminal » (2010: 75). De la même façon, c'est l'association de la voyelle /o/ -laquelle peut également se voir attribuer le statut de cognème ${ }^{37}-$ et du cognème $\mathrm{R}$ qui permet de donner naissance au suffixe -or des noms d'agent et d'instrument. On le voit, la lecture cognématique relève de la recherche d'opérateurs d'amorçage du sens, dans une optique transparadigmatique, quand la lecture morphologique relève déjà de la sémantique et de l'assignation catégorielle de chacune des unités, l'une et l'autre lecture ne s'excluant pas. Si -r-, morphème de futur dont nous venons de proposer une lecture cognématique, apparaît à toutes les formes du futur, il faut encore faire quelques observations sur ce qui caractérise les formes spécifiquement irrégulières de ce paradigme à la lumière d'autres affinités sémiologiques, notamment celles qu'elles présentent avec les formes de l'impératif.

50 En renonçant à la lecture de l'infinitif dans la sémiologie du futur dans l'optique synchronique qui est la nôtre, nous avons précédemment établi un parallèle sémiologique entre l'impératif et le futur pour toutes les formes, parmi lesquelles nous avons isolé un sous-ensemble de radicaux marqués (i) par la perte de la voyelle thématique et (ii) par un radical qui pouvait éventuellement s'écarter du radical de l'infinitif (dir-, har-) : il s'agit là des formes dites « irrégulières ». 
51 La perte de cette voyelle thématique est également caractéristique des formes irrégulières du mode impératif en espagnol (tutoiement singulier) ${ }^{38}$ : DECIR $(\rightarrow$ di), HACER $(\rightarrow$ haz), IR $(\rightarrow v e)$, PONER $(\rightarrow$ pon), SALIR $(\rightarrow$ sal), SER $(\rightarrow$ sé), TENER $(\rightarrow$ ten $)$ et VENIR $(\rightarrow$ ven). Le verbe VALER possède, nous l'avons dit, deux formes pour la deuxième personne du singulier de l'impératif (vale vs val) ${ }^{39}$. Si l'on compare ce paradigme à celui du futur, nous constatons que les mêmes verbes sont concernés par l'irrégularité morphologique, à l'exception des verbes caber, poder, querer, saber, qui sont défectifs :

\begin{tabular}{|c|c|c|c|}
\hline & & FUTUR & Impératif \\
\hline \multirow{2}{*}{$\begin{array}{l}\text { Infinitif à } \\
\text { radical contracté }\end{array}$} & Decir & diré & $d i$ \\
\hline & Hacer & haré & haz \\
\hline \multirow{5}{*}{$\begin{array}{l}\text { Chute de la } \\
\text { prétonique interne }\end{array}$} & Caber & cabré & CABE \\
\hline & Haber & habré & \\
\hline & Poder & podré & PUEDE \\
\hline & Querer & querré & QUIERE \\
\hline & Saber & sabré & SABE \\
\hline \multirow{5}{*}{$\begin{array}{l}\text { Chute vocalique et } \\
\text { épenthèse consonantique }\end{array}$} & Poner & pondré & pon \\
\hline & Salir & saldré & sal \\
\hline & Tener & tendré & ten \\
\hline & Valer & valdré & val/VALE \\
\hline & Venir & vendré & ven \\
\hline \multirow{6}{*}{$\begin{array}{l}\text { Pas d'irrégularité au futur } \\
\text { (contraintes phonétiques diverses) }\end{array}$} & Ir & IRÉ & ve \\
\hline & Ser & SERÉ & sé \\
\hline & Dar & DARÉ & $\mathrm{DA}$ \\
\hline & Estar & ESTARÉ & ESTÁ \\
\hline & Traer & TRAERÉ & TRAE \\
\hline & -ducir & -DUCIRÉ & -DUCE \\
\hline
\end{tabular}

Table 3. Comparaison des morphologies du futur et de l'impératif

Ce constat nous permet d'affirmer deux choses. La première, c'est que le radical est protégé dans les irrégularités du futur, comme dans celles de l'impératif et ce, sous une forme strictement identique dans les cas particuliers de di- et ha-. En outre, les formes marquées par le sceau de l'irrégularité sont sensiblement les mêmes, ce qui confirme 
notre hypothèse selon laquelle il s'agit de formes plus grammaticales : d'un point de vue sémiologique encore une fois, les formes d'impératifs dénuées de voyelles thématiques sont premières en ce qu'elles se présentent sous la forme d'un radical, "à nu », sans l'ajout d'une quelconque désinence ${ }^{40}$.

\section{Bilan}

Une lecture strictement synchronique des formes irrégulières de futur en espagnol nous a permis d'associer à ces formes morphologiquement marquées par l'absence de voyelle thématique -le radical et le morphème - $r$ - de futur restent protégés- un faisceau de comportements sémantico-syntaxiques, tendanciellement cumulatifs mais non obligatoires, qui nous invitent à en réaffirmer le caractère "fondamental ", au moins dans la mesure où ces formes jouissent d'un statut à la croisée entre lexique et grammaire. Nonobstant cette visée synchronique, ces observations nous conduisent à avancer que : i) le périmètre des formes irrégulières tend à se réduire de manière congruente avec ces caractéristiques sémantico-syntaxiques, et: ii) les paradigmes irréguliers invitent à reconnaître dans toutes les formes de futur le cognème $\mathrm{R}$, qui agit dans d'autres réseaux transparadigmatiques de la langue espagnole, et expliquerait en partie que la lecture d'infinitif désormais devenue impossible ne constitue pas un frein au maintien des formes irrégulières dans ce réseau.

\section{BIBLIOGRAPHIE}

ALCOBA, S., 1992, « ¿Consideración no diacrónica de la morfología y acento del tema de futuro? », dans J. A. Bartol Hernández, J. F. García Santos et J. De Santiago Guervós (eds.), Estudios filológicos en homenaje a Eugenio Bustos Tovar, Salamanque, Ediciones Universidad (Acta Salmanticensia, Estudios filológicos, 250) : 35-49.

AZZOPARDI, S., 2011, Le Futur et le Conditionnel : valeur en langue et effets de sens en discours. Analyse contrastive espagnol/français. Thèse en sciences du langage, Université Paul-Valéry - Montpellier 3. BÉNABEN, M., 2002, Manuel de linguistique espagnole (1993), Paris, Ophrys.

BLESTEL, É., 2015, « Quand le plus-que-parfait persiste et signe », Cahiers de Praxématique 64 (en ligne : <https://praxematique.revues.org/3967>).

BLESTEL, É. et FONTANIER, R., 2017, « 'Robó taxi de una parada y chocó por une columna'. Una hipótesis explicativa sobre el empleo de la preposición por en Paraguay », dans A. Palacios Alcaine (coord.), Variación y cambio lingüistico en situaciones de contacto, Madrid, Iberoamericana : 185-204.

BLESTEL, É. et FORTINEAU-BRÉMOND, C., 2015, « La linguistique du signifiant : fondements et prolongements », Cahiers de Praxématique 64 (en ligne : <https://praxematique.revues.org/3967>). 
BOTTINEAU, D., 2004, « Le problème de la négation et sa solution dans la langue anglaise : le cognème N », dans C. Delmas et L. Claude Roux (éds), La contradiction en anglais. Saint-Étienne, Publications de l'université de Saint-Étienne : 27-53.

BOTTINEAU, D., 2007, « The Cognemes of the Spanish Language : towards a Cognitive Modelization of the Submorphemic Units in the Grammatical Words of the Spanish Language », The Public Journal of Semiotics I, $2: 50-74$.

BOYÉ, G., 2000, Problèmes de morpho-phonologie verbale en français, en espagnol et en italien. Thèse de linguistique, Université Paris 7 - Denis-Diderot, UFR de linguistique.

CHEVALIER, J.-C. ET DELPORT, M.-F., 2001, « Propositions pour l'analyse des périphrases verbales ", dans F. Tollis (éd.), La Locution et la Périphrase, du lexique à la grammaire. Paris, L'Harmattan : 115-139.

COMPANY COMPANY, C., 1985, «Los futuros en el español medieval : sus orígenes y su evolución », Nueva Revista de Filología Hispánica XXXIV, 1 : 48-107.

CREA : Corpus de Referencia del Español Actual (en ligne, par : <www.rae.es>).

DARBORD, B. et POTTIER, B., 2008, La Langue espagnole. Grammaire historique. Paris, Armand Colin. DPD : Diccionario Panhispánico de Dudas (en ligne, par : <www.rae.es>).

DRAE : Diccionario de la Real Academia Española (en ligne, par : <www.rae.es>).

FORTINEAU-BRÉMOND, C., 2016, « El continuum léxico/gramática : una alternativa enactiva », dans Á. López García-Molins et D. Jorques Jiménez (éds), Enacción y léxico, Valence, Tirant Humanidades : 97-119.

FORTINEAU-BRÉMOND, C., 2018, « Sur le cognème O en espagnol : quelques propositions », Chréode 2 : 291-310.

FRATINI, V., ACHA J. et LAKA, I., 2014, « Frequency and morphological irregularity are independent variables. Evidence from a corpus study of Spanish verbs », Corpus Linguistics and Linguistic Theory $10: 289-314$

GRANVIK, A., 2009, « Doy, estoy, hay, soy y voy: la combinación atípica de cinco monosílabos con una terminación extraparadigmática. Estado de la cuestión », dans T. Bastardín Candón, M. Rivas Zancarrón et J. Ma García Martín (éds), Estudios de historiografía lingüística, Cadix : Universidad de Cádiz : 307-332.

GUILLAUME G., 1987, Leçons de linguistique de Gustave Guillaume, 1945-1946, série A, Esquisse d'une grammaire descriptive de la langue française IV, publiées sous la direction de R. Valin, W. Hirtle et A. Joly, Québec, Presses de l'Université Laval et Lille, Presses Universitaires de Lille.

HARRIS, J. W., 1969, Spanish Phonology, Cambridge (Mass.), MIT Press.

HARRIS, J. W., 1987, « The Accentual Patterns of Verbs Paradigms in Spanish », Natural Language and Linguistic Theory 5 : 61-95.

HERCE CALLEJA, B., 2016, «Why frequency and morphological irregularity are not independent variables in Spanish », Corpus Linguistics and Linguistic Theory 12 : 389-406.

LAUNAY, M., 1980, « Acerca de los auxiliares y frases verbales », Lingüística española actual II, 1, Madrid : 39-79.

LAUNAY, M., 2003, « Note sur le dogme de l'arbitraire du signe et ses possibles motivations idéologiques », Mélanges de la Casa de Velázquez XXXIII, 2 : 275-284. 
LE TALLEC-LLORET, G., 2012, « Linguistique du signe, linguistique du signifiant : de Mo.La.Che à la cognématique », dans G. Luquet (éd.), Morphosyntaxe et sémantique espagnoles. Théories et applications, Paris, Presses de la Sorbonne Nouvelle : 15-38.

LUQUET, G., 2000a, Regards sur le signifiant, Paris, Presses de la Sorbonne Nouvelle.

LUQUET, G., 2000b, « Une autre façon de concevoir l'histoire de la forme en -ra : un seul signe de langue, plusieurs modes d'emploi à travers les siècles ", dans A. Résano (dir.), Linguistique hispanique Nantes 1998, Nantes, CRINI - Université de Nantes : 101-110.

LUQUET, G., 2004, La teoría de los modos en la descripción del verbo español. Un nuevo planteamiento. Madrid, Arco/Libros.

LUQUET, G., 2010, « De l'iconicité des morphèmes grammaticaux en espagnol », dans G. Le TallecLloret (éd.), Vues et contrevues. Actes du XII ${ }^{e}$ Colloque international de linguistique ibéro-romane. Université de Haute-Bretagne - Rennes 2, 24-26 septembre 2008, Limoges, Lambert-Lucas : 73-83.

MARTÍN VEGAS, R. A., 2014, Los verbos irregulares del español. Clasificacíon de modelos. Madrid, Biblioteca Nueva.

MOLHO, M., 1983, « Del significante verbal en español », Serta Philologica F. Lázaro Carreter, Madrid : 391-411.

MOLHO, M., 1992, « Deux morphogénies : fr. être, esp. ser », Cahiers de linguistique hispanique médiévale XVII, $1: 5-31$.

MOLHO, M., 1997, « Del futuro español y de su historia », Analecta Malacitana XX, $1:$ 267-278.

NOBILE, L., 2015, « Phonemes as images: an experimental inquiry into shape-sound symbolism applied to the distinctive features of French », dans M. K. Hiraga et al. (éds), Iconicity : East Meets West, Amsterdam, John Benjamins : 71-91.

PAGÈS, S., 2012, « Architecture du système verbal espagnol actuel : description et analyse du jeu du signifiant », Studia UBB Philologia LVII, 3 : 41-56.

PAGÈS, S., 2017, « La défectivité verbale comme "problème" de linguistique : application à la langue espagnole (description, implications, analyse) », conférence (n. p.) du 9 mars 2017 au séminaire RELIR (EA 4080 / Université Paris-Sorbonne, UFR Études ibériques et latinoaméricaines).

PÉREZ SALAZAR, C., 2002, « Futuro y condicional en español moderno : variación y variedad a lo largo de dos siglos ", dans C. Saralegui Platero et M. Casado Velarde (éds), Pulchre, Bene, Recte. Estudios en homenaje al prof. Fernando González Ollé. Pampelune, EUNSA : 1081-1103.

PENNY, R., 2002, A History of the Spanish Language, Cambridge, Cambridge University Press.

POTTIER, B., 1961, « Sobre el concepto de verbo auxiliar », dans B. Pottier, Lingüística moderna y filología hispánica [trad. espagnole de M. Blanco Álvarez], Madrid, Gredos : 194-203.

QUITARD, M., 2010, Recherche sur la semi-auxiliarité en espagnol : le cas de ir, Thèse de doctorat, Paris, Université Sorbonne Nouvelle.

RAMACHANDRAN, V. S. et HUBBARD, E. M., 2001, « Synaesthesia - a window into perception, thought, and language ", Journal of Consciousness Studies 8(12) : 3-34.

REAL ACADEMIA ESPAÑOLA, 1973, Esbozo de una nueva gramática de la lengua española, Madrid, Espasa-Calpe.

RINI, J., 1995, « Syntactic and Pragmatic Factors in the Morphological Reduction of Latin HABEO > Spanish (h)e », Neophilologus LXXIX : 421-432. 
SARALEGUI PLATERO, C., 1983, « Morfología del futuro y condicional castellanos : polimorfismo antiguo y fijación lingüística », Medioevo Romanzo VIII, 3 : 419-459.

SCHMIDELY, J., 1988, « La -y de doy, estoy, soy, voy », dans M. Ariza, A. Salvador, A. Viudas (éds), Actas del I Congreso internacional de Historia de la lengua española, Madrid, Arco/Libros : 611-619.

SCHMIDELY, J., 1996, « La -Y de hay », dans A. Alonso González, L. Castro Ramos, B. Gutiérrez Rodilla et J. A. Pascual Rodríguez (éds), Actas del III Congreso internacional de historia de la lengua española, Madrid, Arco/Libros : 195-204.

TOLEDO, Á. E., 2013, Histoire thématique et descriptive du double paradigme des participes passés en espagnol. Thèse en sciences du langage. Paris, EHESS.

TRAUGOTT, E. et HEINE, B. (éds), 1991, Approaches to Grammaticalization, Amsterdam, John Benjamins.

VATRICAN, A., 2016, El Condicional en español, Madrid, Arco/Libros.

ZALIO, D., 2013, Étude synchronique contrastive des descendants romans d'ESSE et de STARE : les signifiants italiens essere et stare à la lumière des signifiants espagnols ser et estar. Thèse de linguistique romane, Université Paris-Sorbonne.

\section{NOTES}

1. Voir p.ex., pour l'espagnol et le français, la bibliographie d'Azzopardi 2011. Ou, sur le conditionnel espagnol et d'un tout autre point de vue théorique, le travail de Vatrican 2016.

2. Entre autres à la faveur de discussions entre les participants à l'opération « Le futur dans les langues romanes » (EA 7345 CLESTHIA).

3. Toutes choses égales d'ailleurs, les formes irrégulières du conditionnel, en tant que, dans les faits, strictement coextensives aux mêmes verbes et présentant les mêmes particularités morphologiques, seront logées à la même enseigne, et il n'est pas question pour nous de les traiter séparément sous ce rapport : dire ici « futur », ce sera entendre « futur-et-conditionnel ».

4. Même si certains futurs irréguliers concernent des verbes flexionnellement déviants par ailleurs, tels DAR 'donner', justement (fut. rég. daré 'je donnerai’ mais prés. irr. Doy 'je donne', alors que pour CANTAR 'chanter' on a resp. cantaré / canto, tous deux réguliers).

5. Même remarque que dans la note précédente, par exemple pour IR 'aller' (également invoqué dans Boyé 2000).

6. Sauf à considérer les formes réputées "contractées" (ou "syncopées ", selon les termes anciens de Correas) de type HACER 'faire' $\rightarrow$ haré 'je ferai' et DECIR 'dire' $\rightarrow$ diré 'je dirai' comme des cas de changement de radical, donc de "thème» de conjugaison. En réalité, les bases infinitives far et dir sont non seulement quasi-pan-romanes, mais bien attestées en espagnol médiéval (Luquet 2000a: 62-63). Nous les appellerons « infinitifs à radical contracté » pour les distinguer des formes résultant de la seule chute de la voyelle prétonique interne.

7. Malgré les aperceptions originales et les positions aussi hardiment qu'opiniâtrement défendues par Lanly, on ne discutera pas ici l'hypothèse de la formation des futurs et conditionnels romans, notamment espagnols, telle que décrite depuis Diez comme résultant d'une agglutination de ce type. Voir p. ex. comment Molho (1997 : publication post-mortem d'un inédit assez ancien) réfute Lanly depuis une perspective psychosémantique.

8. En espagnol contemporain, HABER 'avoir' n'apparaît que dans des emplois d'auxiliaire dans les temps composés ou dans des emplois impersonnels (p. ex. he cantado 'j'ai chanté', había mucha gente 'il y avait beaucoup de monde'). 
9. Real Academia Española 1973 : 256, n. 4 : «Lo mejor es explicar históricamente la formación del futuro y el condicional, [...] sin entrar en pormenores » [Nous traduisons]; on sait que les contenus de morphologie y sont attribuables à Salvador Fernández Ramírez.

10. Ce qui est de bonne guerre : le rêve, en somme, qui œuvre au principe de toute élaboration d'une grammaire.

11. Ici à partir du modèle d'Alcoba (1992), légèrement modifié par nous.

12. VT : voyelle thématique; MT : morphème modo-temporel. Commentaires $:$ i) on peut imaginer d'agglutiner MT et Dés.Pers de telle sorte qu'ils ne constituent plus qu'une seule unité intégrée ; ii) la question peut se poser de savoir si le morphème MT ne serait pas seulement temporel, et non modal : en tout cas, à croiser la double opposition intra-paradigmatique des terminaisons P1/P3 et inter-paradigmatique Fut/Cond, les données des formes irrégulières comme régulières restent conformes aux préconisations de Gilles Luquet (2004), qui ne reconnaît que deux modes, respectivement actualisant (caractérisé par dés.P1 $\neq \mathrm{P} 3$ ) et non-actualisant (où dés.P1 = P3); iii)

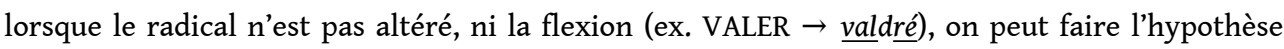
purement phonotactique d'un simple problème fort courant de sandhi au niveau de VT (ici par découplage articulatoire : dénasalisation de la fin du segment nasal).

13. Le courant théorique connu comme " la linguistique du signifiant », initiée par J.-C. Chevalier, M. Launay et M. Molho au milieu des années quatre-vingt a inspiré de nombreux travaux chez les hispanistes ces trente dernières années. Cette approche, qui se fonde sur les principes d'unicité et de bi-univocité du signe linguistique, accorde une place de première importance à la dimension iconique du langage. Voir à ce sujet Blestel et Fortineau-Brémond (2015).

14. Voir notamment Darbord et Pottier (2008), Bénaben (2002), Luquet (2000a), Molho (1992).

15. Sauf TRAER 'apporter' et, a fortiori, ses composés préfixés, qui, après avoir abandonné la variante médiévale -troxe (étymologique < lat. *TRAXUI) au bénéfice d'un réalignement analogique « interne » sur -truje selon cette logique de réduction des voyelles radicales, se sont stabilisés aujourd'hui en -traje par une analogie plus « régularisante » en ce qu'elle retrouve sa voyelle radicale d'infinitif (et de ses autres paradigmes) : si TRAER ne manque pas d'emplois (surtout locutionnels) de type attributif (me trae sin cuidado 'ça me laisse indifférent', me trae loco 'ça me rend fou' etc.), il est, dans la plupart de ses emplois, l'un des moins « fondamentaux » de cette nomenclature des verbes à prétérit irrégulier.

16. Voir notamment Molho 1983 ; Schmidely 1988 et 1996, Luquet 2000a : 61-70.

17. Luquet $2000 \mathrm{a}: 56$.

18. La voyelle thématique $a$ du prétérit pourtant irrégulier de TRAER le désigne comme tendanciellement aux marges de ce sous-ensemble : cf. supra notre n. 15.

19. C'est la représentation qu'en fournit Martín Vegas 2014: 74. Toutes les sources ne manifestent pas la même opinion: le DPD ne stigmatise par exemple pas les formes telles que DESDECIR $\rightarrow$ desdiré ; au contraire, il les présente même hiérarchiquement en premier lieu, mais accorde le bénéfice de la «normalité dans certaines zones » aux «formes régulières » desdeciré etc. Il ne fait du reste en cela aucune différence (à l'inverse de Martín Vegas) dans le traitement de DESDECIR et de CONTRADECIR. Pour ce qui est des préfixés sur VALER, Martín Vegas assigne à EQUIVALER un fut. irr. equivaldrá sur le modèle de sa base, mais considère comme régulier PREVALER ( $\rightarrow$ prevalerá) : en réalité, le très faible « rendement » paradigmatique (pour le dire en ses propres termes) de cet infinitif dans l'usage, s'accompagne de sa forte mise en concurrence avec le verbe à infixation inchoative PREVALECER (fut. rég. $\rightarrow$ prevalecerá), et l'on a sans doute affaire ici, sinon à une « variation » de groupe (comme dans ital. AMMOLLIRE/AMMOLLARE), du moins à une « variation » de modèle.

Pour une nomenclature indicative des lexèmes verbaux anciens et modernes en cause, voir Penny (2002 § 3.7.7.4), Company Company 1985 ou Martín Vegas 2014. Le débat sur la corrélation entre fréquence et irrégularité formelle s'est récemment relancé entre Fratini, Acha \& Laka 
(2014) et Herce Calleja (2016), en particulier sur la prise en considération des dérivés affixaux et de leur comportement en corpus.

20. À noter que le verbe VALER présente deux formes pour la deuxième personne du singulier de l'impératif : une forme régulière vale, et une forme irrégulière val, qui n'est plus usitée. $C f$. infra $\S 7$.

21. C'était déjà là la proposition de G. Guillaume pour la langue française (Guillaume 1987 : Leçon du 12 décembre 1946, série A).

22. C'est aussi la position de Chevalier et Delport (2001:128).

23. Cette exception montre que cette caractéristique n'est pas obligatoire pour que ces formes puissent prétendre au statut de verbes « marqués » relativement aux autres. Voir infra.

24. Exemples extraits du CREA. Hiérarchie des acceptions ou emplois dans DRAE.

25. Exemples extraits du CREA.

26. Exemples extraits du CREA.

27. En position initiale, le groupe /sifflante + bilabiale, dentale ou vélaire/ est rejeté en espagnol (le problème plus général de l'extrasyllabicité de l'esse impura en roman reçoit diverses réponses systémiques selon les langues).

28. Le fait que la désinence en yod affecte les $1^{\text {ères }}$ personnes des verbes concernés (ou la "personne univers" de hay) est bien au cœur des discussions sur son origine : voir à ce sujet Schmidely 1988 et 1996, ainsi que le point méta-théorique proposé par Granvik 2009.

29. Voir Fortineau-Brémond (2016: 97).

30. Par exemple avec Traugott et Heine (1991:1) au sujet de la grammaticalisation: "Grammaticalization is the linguistic process, both through time and synchronically, of organization of categories and of coding. The study of grammaticalization therefore highlights the tension between relatively unconstrained lexical expression and more constrained morphosyntactic coding, and points to relative indeterminacy in language and to the basic nondiscreteness of categories. "

31. Nous entendons par "submorphème " tout élément lexical ou grammatical qui, en-deçà du morphème, est considéré comme jouant un rôle dans l'amorçage du sens.

32. On peut considérer la cognématique comme l'une des fort nombreuses théories visant à étudier les dispositifs et le fonctionnement de l'iconicité lato sensu dans les langues, en l'occurrence au niveau grammatical autant que lexical. Pour d'autres propositions ou propositions de validation expérimentale des faits d'iconicité, voir p. ex. Nobile 2015 ou Ramachandra \& Hubbard 2001.

33. On aura compris que ce lien entre $R$ et agentivité est ici envisagé en première instance comme ne concernant que l'espagnol. Par ailleurs, l'agentivité dont il est question est très précisément celle qui est spécifiquement liée à la présence de ce cognème.

34. On pourrait faire l'hypothèse que ce processus de réanalyse sémiologique et sémantique est également à l'origine de la métathèse de la préposition latine PRŌ (+ ablatif) devenue esp. por, par analogie avec PER (+ accusatif). Sur l'étymon de por, voir Darbord et Pottier (2008: 203).

35. Cette dernière forme est traditionnellement étiquetée comme un «subjonctif imparfait de l'indicatif » mais elle fait l'objet de nombreuses controverses. Voir à ce sujet Luquet (2000b).

36. Si l'on s'intéresse aux propriétés articulatoires du phonème qui réalise le cognème $T$, nous pouvons constater que nous avons affaire à une occlusive -ou une affriquée dans le cas des participes dits « irréguliers » de type dicho- (sourde ou sonore) qui implique par conséquent une obstruction du flux d'air dans le canal vocal par le contact entre la langue et les dents inférieures ou supérieures. Ce mode et ce point d'articulation entrent par iconicité dans ce qui constitue la valeur du cognème $\mathrm{T}$, à savoir, l'atteinte d'un seuil, d'une borne. C'est la raison pour laquelle l'opposition de sonorité n'est pas pertinente et que ce que nous désignons par cognème $\mathrm{T}$ peut inclure également des signifiants contenant le phonème dental sonore /d/ comme les participes. 37. Voir la proposition de Fortineau-Brémond (2018) sur ce sujet. 
38. C'est le cas en dehors des zones de voseo, dans lesquelles la voyelle thématique est rétablie à l'impératif.

39. Deux formes dont on peut cependant douter de la productivité à l'impératif. Sur la question de la « défectivité » de certains paradigmes, sans doute liée à celle de l'irrégularité et intéressant à la fois propriétés des verbes et des tiroirs verbaux concernés, voir le travail en cours de Pagès (2017).

40. Ce constat inviterait par ailleurs à en faire une analyse plus fine du point de vue de leur structuration cognématique : on peut d'ores et déjà se demander si l'opposition haz / haré n'est pas la manifestation de l'opposition cognématique $\mathrm{R}$ d'impulsion' / $\mathrm{S}$ de 'continuation' mise en lumière par Luquet (2010). De même la position finale de $/ \mathrm{n} /$ dans les formes pon, ven et ten pourrait-elle être vue comme l'actualisation du cognème $\mathrm{N}$ d'intériorité'. Ce sont là des hypothèses qui mériteraient d'être explorées.

\section{RÉSUMÉS}

Les futurs et conditionnels irréguliers de l'espagnol contemporain ont peu été étudiés en synchronie du point de vue explicatif. La notable tentative morphosémantique de Luquet (2000a) mérite cependant d'être poursuivie au-delà de ses propres réticences, si l'on veut comprendre à la fois la cohérence raisonnée du sous-système marqué qu'ils constituent et le système paradigmatique des formes de futurs dans son ensemble. Morphème $d$ '\{infinitif\} , $r$ se laisse ainsi non seulement réanalyser en - $r$ - morphème de \{futur\}, mais peut également être lu (notamment à la suite de Bottineau 2007 et Luquet 2010) à l'échelle sub-morphémique comme cognème $\mathrm{R}$ d' « impulsion ».

Irregular futures and conditionals in contemporary Spanish have been scarcely studied from an explicative synchronic point of view. Nevertheless, Luquet's noteworthy morphosemantic attempt (2000a) should be continued beyond his own reticences in order to understand both the reasoned coherence of the marked sub-system they make up and the paradigmatic system of future forms as a whole. Thus not only \{infinitive\} morpheme $-r$ is likely to be reanalysed as a \{future\} morpheme -r-, but it may also be considered (following Bottineau 2007 and Luquet 2010 among others) at a sub-morphemic scale as a R cogneme for 'impulsion'.

\section{INDEX}

Mots-clés : temps futur irrégulier, espagnol, cognème

Keywords : irregular future tense, Spanish, cogneme

\section{AUTEURS}

\section{ERIC BEAUMATIN}

Université de la Sorbonne Nouvelle - Paris 3 / EA 7345 CLESTHIA 


\section{ÉLODIE BLESTEL}

Université de la Sorbonne Nouvelle - Paris 3 / EA 7345 CLESTHIA 